\title{
Anti-EGFRvIII Immunotoxin MR1-1
}

National Cancer Institute

\section{Source}

National Cancer Institute. Anti-EGFRVIII Immunotoxin MR1-1. NCI Thesaurus. Code C88307.

A recombinant immunotoxin consisting of sing le-chain variable domain fragment antibody directed ag ainst the tumor-specific antigen EGFRvIII (MR1scFv) fused to domains II and III of the Pseudomonas exotoxin (PE38KDEL), with potential antineoplastic activity. Upon administration, the antibody moiety of anti-EGFRvIII immunotoxin MR1-1 binds to EGFRvIII; upon internalization, the exotoxin portion inhibits protein synthesis, resulting in a reduction in tumor cell proliferation of EGFRvIIIexpressing tumor cells. EGFRvIII, a type III in-frame deletion mutation of the epidermal growth factor receptor (EGFR) gene, is expressed by a variety of cancers, including glioblastoma multiforme, non-small lung carcinoma, and breast carcinoma. Compared to intact IgG antibodies, single-chain antibodies such as MR1scFv are smaller and may penetrate tumors better. Pseudomonas exotoxin PE38KDEL was modified to remove the natural cell binding domain. 